\title{
Dispersive Gap Mode of Phonons in Anisotropic Superconductors
}

\author{
Masanori ICHIOKA* and Kazushige MACHIDA \\ Department of Physics, Okayama University, Okayama 700-8530
}

(Received April 4, 2003 )

\begin{abstract}
We estimate the effect of the superconducting gap anisotropy in the dispersive gap mode of phonons, which is observed by the neutron scattering on borocarbide superconductors. We numerically analyze the phonon spectrum considering the electron-phonon coupling, and examine contributions coming from the gap suppression and the sign change of the pairing function on the Fermi surface. When the sign of the pairing function is changed by the nesting translation, the gap mode does not appear. We also discuss the suppression of the phonon softening of the Kohn anomaly due to the onset of superconductivity. We demonstrate that observation of the gap dispersive mode is useful for sorting out the underlying superconducting pairing function.
\end{abstract}

KEYWORDS: gap mode in phonons, anisotropic superconducting gap, electron-phonon coupling, borocarbide.

\section{Introduction}

In the materials with strong electron-phonon interactions, we can obtain the information of the electronic state by investigating phonon properties. The Kohn anomaly of the phonon dispersion comes from the nesting features of the band structure in simple metals via electron-phonon interaction. ${ }^{1)}$ It is known that the gap mode of phonons appears at $\omega=2 \Delta$ in the superconducting state with a gap $\Delta$. In $2 \mathrm{H}-\mathrm{NbSe}_{2}$, the Raman scattering $^{2)}$ observes the gap mode around zero phonon wave vector $\boldsymbol{q}=0$. The gap mode at $\boldsymbol{q} \neq 0$ is reported by the neutron scattering on $\mathrm{YNi}_{2} \mathrm{~B}_{2} \mathrm{C} .{ }^{3-5)}$ There, the phonon dispersion shows the Kohn anomaly at the nesting wave vector $\left.\boldsymbol{Q} \sim \pi(0.55,0,0),{ }^{6}\right)$ and the new peak of the dispersive gap mode appears below the original phonon dispersion around $\boldsymbol{q}=\boldsymbol{Q}$. The neutron scattering on $\mathrm{LuNi}_{2} \mathrm{~B}_{2} \mathrm{C}$ reports that the peak energy at the phonon dispersion is shifted to lower energy and that the peak intensity is enhanced below the superconducting transition temperature $T_{\mathrm{c}}{ }^{7}{ }^{7}$ Theoretically, the gap mode is obtained by considering the contribution of the electron-phonon coupling in the phonon selfenergy. ${ }^{8-11)}$ However, previous theoretical works have investigated in the isotropic $s$-wave superconductivity case, and they have not considered the dispersive behavior of the gap mode.

Band calculations suggest that borocarbide superconductors are conventional BCS superconductors with the pairing interaction mediated by the electron-phonon interaction. ${ }^{12-15)}$ However, the direct observation of the superconducting gap of $\mathrm{YNi}_{2} \mathrm{~B}_{2} \mathrm{C}$ by photoemission spectroscopy reports that the gap has large anisotropy and that the minimum of the gap seems to be zero as if it has point or line nodes. ${ }^{16)}$ And the impurity effects suggest that they are extreme anisotropic $s$-wave superconductors. This gap structure is also supported by the specific heat measurement. ${ }^{17,18)}$ Thermal conductivity experiment by rotating magnetic field suggests that the superconducting gap has point nodes. ${ }^{19)}$ Therefore, it is important to study how the superconducting gap anisotropy affects the dispersive gap mode of phonons.

\footnotetext{
* E-mail address: oka@mp.okayama-u.ac.jp
}

In this paper, we investigate the effect of the gap anisotropy on the gap mode of phonons by calculating the dispersion and intensity of the gap mode around $\boldsymbol{q}=\boldsymbol{Q}$. As the gap anisotropy effect, we examine the case when the superconducting gap is suppressed around the node points, and the case when the order parameter changes the sign by the nesting translation on the Fermi surface as in the $d$-wave pairing. The phonon properties are studied by varying $2 \Delta$ from the weaker case to the stronger case relative to the phonon frequency. And we discuss the experimental data observed in $\mathrm{YNi}_{2} \mathrm{~B}_{2} \mathrm{C}$ and $\mathrm{LuNi}_{2} \mathrm{~B}_{2} \mathrm{C}$. To obtain the renormalized phonon Green's function in the random phase approximation (RPA), we calculate the dynamical charge susceptibility in anisotropic superconductors. In the calculation, we use the electron dispersion characterized by the nesting vector $\boldsymbol{Q} \sim \pi(0.55,0,0)$, which reproduce the Kohn anomaly. When the Kohn anomaly is strong, the phonon is softened at $\boldsymbol{q} \sim \boldsymbol{Q}$, indicating the transition to the charge density wave (CDW) state. This softening is suppressed by the onset of superconductivity.

After explaining our formulation in $\S 2$, we present our results of the phonon spectral function and discuss the effect of anisotropic superconductivity in $\S 3$. The suppression of the phonon softening is discussed in $\S 4$. The last section is devoted to summary and discussions.

\section{Formulation}

As the Fermi surface shape of borocarbide superconductors is complicated, ${ }^{6,15)}$ we simplify the dominant large Fermi surface to the simple two-dimensional Fermi surface which reproduces the nesting property of nesting vector $\boldsymbol{Q}=\left(Q_{x}, 0,0\right) \sim \pi(0.55,0,0)$ as in the borocarbides. Then, we use the following dispersion of the electrons,

$$
\begin{aligned}
K_{\boldsymbol{k}}= & -2 t\left(\cos k_{x}+\cos k_{y}\right)-4 t^{\prime} \cos k_{x} \cos k_{y} \\
& -2 t^{\prime \prime}\left(\cos 2 k_{x}+\cos 2 k_{y}\right)-\mu
\end{aligned}
$$

with the transfer between nearest $(t)$, second nearest $\left(t^{\prime}\right)$ and third nearest $\left(t^{\prime \prime}\right)$ neighbor sites in two-dimensional square lattice of the basal plane in tetragonal crystal. 
The lattice constant is taken to unity. Here, we set $t=1, t^{\prime}=-0.6, t^{\prime \prime}=0.1$ and the chemical potential $\mu=-1.05$. The energy and temperature are scaled by $t$ throughout this paper. The Fermi surface obtained by $K_{\boldsymbol{k}}=0$ is shown in Fig. 1(a). Since it has flat surface at $k_{x} \sim \pm 0.275 \pi$, it gives good nesting for $\boldsymbol{Q} \sim \pi(0.55,0,0)$.
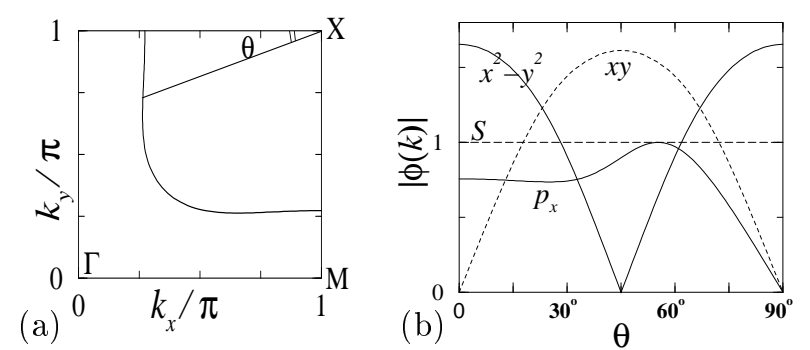

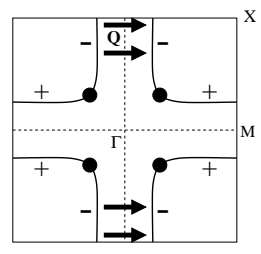

(c) $d_{x^{2}-y^{2}}$

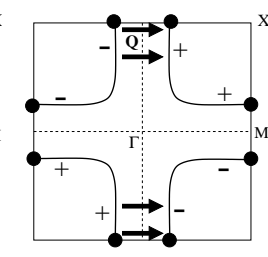

(d) $d_{x y}$

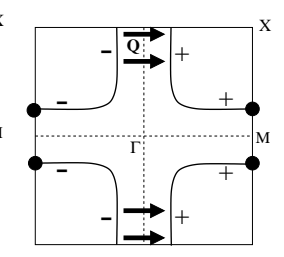

(e) $p_{x}$
Fig. 1. (a) Fermi surface for $t=1, t^{\prime}=-0.6, t^{\prime \prime}=0.1$ and $\mu=-1.05$ in momentum region $0 \leq k_{x} \leq \pi$ and $0 \leq k_{y} \leq \pi$. (b) The gap amplitude of the pairing functions along the Fermi surface. We plot $\left|\phi_{s}(\boldsymbol{k})\right|,\left|\phi_{d_{x^{2}-y^{2}}}(\boldsymbol{k})\right|=\left|\phi_{s_{x^{2}-y^{2}}}(\boldsymbol{k})\right|,\left|\phi_{d_{x y}}(\boldsymbol{k})\right|=$ $\left|\phi_{s_{x y}}(\boldsymbol{k})\right|$, and $\left|\phi_{p_{x}}(\boldsymbol{k})\right|$ as a function of $\theta$, which is the angle centered at the X point as shown in (a). The lower panels schematically present the sign of the pairing function along the Fermi surface for the $d_{x^{2}-y^{2}}$-wave (c), the $d_{x y}$-wave (d) and the $p_{x^{-}}$ wave (e) cases in the region $-\pi \leq k_{x} \leq \pi$ and $-\pi \leq k_{y} \leq \pi$. The solid circles on the Fermi surface present the node points of the pairing functions. Arrows show the nesting vector $\boldsymbol{Q}$.

As for the pairing function $\phi(\boldsymbol{k})$, we use $\phi_{s}(\boldsymbol{k})=1$ for isotropic $s$-wave pairing, $\phi_{d_{x^{2}-y^{2}}}(\boldsymbol{k})=\cos k_{x}-\cos k_{y}$ for $d_{x^{2}-y^{2}}$-wave, and $\phi_{d_{x y}}(\boldsymbol{k})=2 \sin k_{x} \sin k_{y}$ for $d_{x y^{-}}$ wave. The gap amplitude along the Fermi surface is shown in Fig. 1(b). The flat part of the Fermi surface related to the nesting is ranged for $0 \leq \theta<30^{\circ}$. At $\theta=0,\left|\phi_{d_{x^{2}-y^{2}}}(\boldsymbol{k})\right|$ has large gap, and $\left|\phi_{d_{x y}}(\boldsymbol{k})\right|=0$ due to the node structure. With increasing $\theta,\left|\phi_{d_{x^{2}-y^{2}}}(\boldsymbol{k})\right|$ $\left(\left|\phi_{d_{x y}}(\boldsymbol{k})\right|\right)$ is decreased (increased). These gap dependences on $\theta$ are averaged along the flat Fermi surface when they contribute to the phonon properties.

It is also important to see the sign change of the pairing function through the nesting translation $\boldsymbol{Q}$. In the $d_{x^{2}-y^{2} \text {-wave case, there are nodes along the diagonal }}$ lines $k_{y}= \pm k_{x}$. In the $d_{x y}$-wave case, nodes are located along the horizontal lines $k_{y}=0, \pm \pi$ and the vertical lines $k_{x}=0, \pm \pi$. The sign of $\phi_{d_{x^{2}-y^{2}}}(\boldsymbol{k})$ is the same at the both ends connected by the nesting $\boldsymbol{Q}$, as schematically shown in Fig. 1(c). But, the sign of $\phi_{d_{x y}}(\boldsymbol{k})$ changes by the nesting $\boldsymbol{Q}$, as shown in Fig. 1(d).

For extreme anisotropic $s$-wave superconductors, we use $\phi_{s_{x^{2}-y^{2}}}(\boldsymbol{k})=\left|\phi_{d_{x^{2}-y^{2}}}(\boldsymbol{k})\right|$ and $\phi_{s_{x y}}(\boldsymbol{k})=\left|\phi_{d_{x y}}(\boldsymbol{k})\right|$. We call them as the $s_{x^{2}-y^{2}}$ case and the $s_{x y}$ case, respectively. They have the same superconducting gap as those of the $d_{x^{2}-y^{2}}$-wave and the $d_{x y}$-wave cases. But they do not change the sign of the pairing function under $\pi / 2$-rotation. Then, the pairing function does not change the sign by the nesting $\boldsymbol{Q}$. We also consider the $p_{x}$-wave pairing case $\phi_{p_{x}}(\boldsymbol{k})=\sin k_{x}$, while it is not for the pairing of borocarbide superconductors. The pairing function $\phi_{p_{x}}(\boldsymbol{k})$ has nodes along the vertical lines $k_{x}=0$, $\pm \pi$. As shown in Fig. 1(b), $\phi_{p_{x}}(\boldsymbol{k})$ has large gap at the flat Fermi surface $0 \leq \theta<30^{\circ}$. But, $\phi_{p_{x}}(\boldsymbol{k})$ changes the sign by the nesting $\boldsymbol{Q}$ as shown in Fig. 1(e). Then, we can clarify the effect of the sign change by considering $\phi_{p_{x}}(\boldsymbol{k})$.

First, we calculate the dynamical charge susceptibility $\chi_{n n}(\boldsymbol{q}, \omega)$ in the superconducting state with the superconducting gap $\Delta_{\boldsymbol{k}}=\Delta(T) \phi(\boldsymbol{k})$. This is given by

$$
\begin{aligned}
& \chi_{n n}(\boldsymbol{q}, \omega)=-\frac{1}{2} \sum_{\boldsymbol{k}}\left(1+\frac{K_{\boldsymbol{k}+\boldsymbol{q}} K_{\boldsymbol{k}}-\operatorname{Re}\left\{\Delta_{\boldsymbol{k}+\boldsymbol{q}} \Delta_{\boldsymbol{k}}^{*}\right\}}{E_{\boldsymbol{k}+\boldsymbol{q}} E_{\boldsymbol{k}}}\right) \\
& \times\left(\frac{f\left(E_{\boldsymbol{k}+\boldsymbol{q}}\right)-f\left(E_{\boldsymbol{k}}\right)}{E_{\boldsymbol{k}+\boldsymbol{q}}-E_{\boldsymbol{k}}-\omega}+\frac{f\left(E_{\boldsymbol{k}+\boldsymbol{q}}\right)-f\left(E_{\boldsymbol{k}}\right)}{E_{\boldsymbol{k}+\boldsymbol{q}}-E_{\boldsymbol{k}}+\omega}\right) \\
& -\frac{1}{2} \sum_{\boldsymbol{k}}\left(1-\frac{K_{\boldsymbol{k}+\boldsymbol{q}} K_{\boldsymbol{k}}-\operatorname{Re}\left\{\Delta_{\boldsymbol{k}+\boldsymbol{q}} \Delta_{\boldsymbol{k}}^{*}\right\}}{E_{\boldsymbol{k}+\boldsymbol{q}} E_{\boldsymbol{k}}}\right) \\
& \times\left(\frac{f\left(E_{\boldsymbol{k}+\boldsymbol{q}}\right)+f\left(E_{\boldsymbol{k}}\right)-1}{E_{\boldsymbol{k}+\boldsymbol{q}}+E_{\boldsymbol{k}}-\omega}+\frac{f\left(E_{\boldsymbol{k}+\boldsymbol{q}}\right)+f\left(E_{\boldsymbol{k}}\right)-1}{E_{\boldsymbol{k}+\boldsymbol{q}}+E_{\boldsymbol{k}}+\omega}\right)
\end{aligned}
$$

with $E_{\boldsymbol{k}}=\left(K_{\boldsymbol{k}}^{2}+\left|\Delta_{\boldsymbol{k}}\right|^{2}\right)^{1 / 2}$ and the Fermi distribution function $f(E)$. We perform $\boldsymbol{k}$-summation within a Brillouin zone. The temperature dependence of $\Delta(T)$ is determined from the BCS relation,

$$
1=-V_{\mathrm{s}} \sum_{\boldsymbol{k}} \frac{1-2 f\left(E_{\boldsymbol{k}}\right)}{2 E_{\boldsymbol{k}}}|\phi(\boldsymbol{k})|^{2},
$$

where the pairing interaction $V_{\mathrm{s}}(<0)$ is determined by a given $\Delta(T=0)$.

The neutron-scattering profile of a phonon is approximately the phonon spectral function $S(\boldsymbol{q}, \omega)$, i.e., the imaginary part of the phonon Green's function,

$$
S(\boldsymbol{q}, \omega)=-\operatorname{Im} D(\boldsymbol{q}, \omega) .
$$

The renormalized phonon Green's function $D(\boldsymbol{q}, \omega)$ is given by

$$
D(\boldsymbol{q}, \omega)^{-1}=D_{0}(\boldsymbol{q}, \omega)^{-1}-\Pi(\boldsymbol{q}, \omega) .
$$

In the phonon selfenergy, polarizability is written as

$$
\Pi(\boldsymbol{q}, \omega)=-\left|g_{\boldsymbol{q}}\right|^{2} \chi_{n n}(\boldsymbol{q}, \omega)
$$

in RPA. The bare phonon Green's function

$$
D_{0}(\boldsymbol{q})=\frac{2 \omega_{\boldsymbol{q}}}{\omega^{2}-\omega_{\boldsymbol{q}}^{2}} .
$$

The electron-phonon coupling constant $g_{\boldsymbol{q}}$ and the phonon dispersion $\omega_{\boldsymbol{q}}$ are treated as constants, since we consider narrow $\boldsymbol{q}$-region around $\boldsymbol{Q}$.

\section{Phonon spectral function}

\subsection{Normal state}

In our calculation, we typically set $\left|g_{\boldsymbol{q}}\right|^{2}=0.1, \omega_{\boldsymbol{q}}=$ 0.15 and consider the low temperature case $T=0.001$, where $\Delta(T) \sim \Delta(T=0)$. We present the phonon spec- 
tral function in the normal state $\Delta(T)=0$ in Fig. 2(a). It shows $S(\boldsymbol{q}, \omega)$ along the $q_{x}$-direction around $\boldsymbol{Q}$. By the effect of $\chi_{n n}(\boldsymbol{q}, \omega)$ in the normal state, the phonon dispersion is shifted to lower energy than $\omega_{\boldsymbol{q}}$, and it has a minimum at $\boldsymbol{Q}$ reflecting the nesting properties. The peak width of $S(\boldsymbol{q}, \omega)$ comes from $\operatorname{Im} \chi_{n n}(\boldsymbol{q}, \omega)$. The temperature dependence of the phonon dispersion is weak in this parameter case. The minimum energy of the dispersion is $\omega=0.088$ at $T=0.05$. It slightly decreases to $\omega=0.086$ at $T=0.001$.
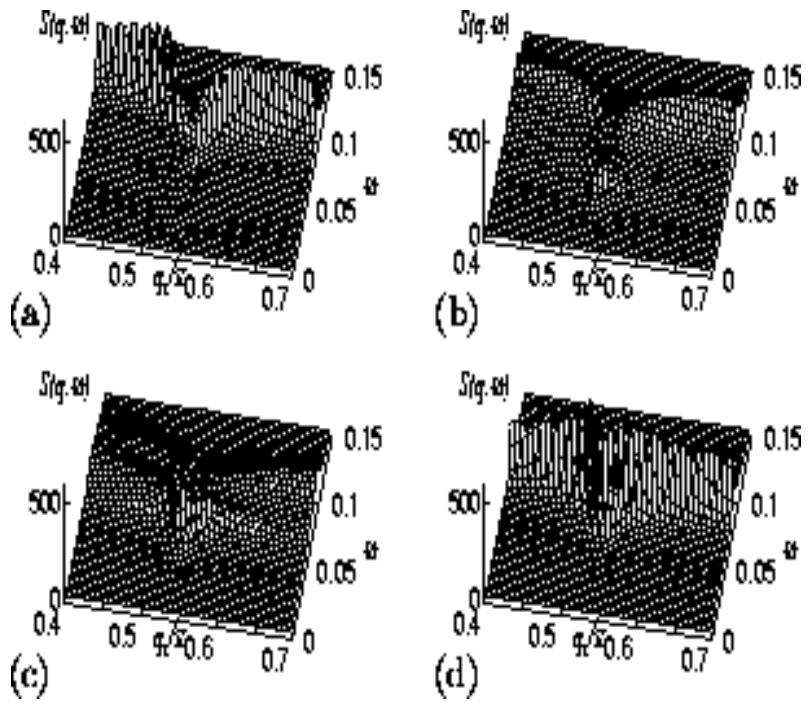

Fig. 2. Phonon spectral function $S\left(\boldsymbol{q}=\left(q_{x}, 0,0\right), \omega\right)$ in the range $0.4 \leq q_{x} / \pi \leq 0.7$ for the normal state $\Delta=0$ (a) and the isotropic $s$-wave superconducting state with $\Delta=0.025$ (b), 0.035 (c) and $0.050(d)$.

When $\omega_{\boldsymbol{q}}$ is further decreased, the phonon dispersion at $\boldsymbol{q} \sim \boldsymbol{Q}$ approaches zero energy on lowering temperature by the Kohn anomaly. This phonon softening case is considered in $\S 4$.

\subsection{Isotropic s-wave superconducting state}

We consider how $S(\boldsymbol{q}, \omega)$ is changed in the superconducting state. First, we investigate the isotropic $s$-wave case. We consider the $\Delta$-dependence of the phonon properties, where $\Delta \equiv \Delta(T=0)$. Figures $2(\mathrm{~b})$-(d) show the change of $S(\boldsymbol{q}, \omega)$ with increasing $\Delta$. When $2 \Delta$ approaches the phonon dispersion from lower energy, the new peak of the gap mode appears below the phonon dispersion, as shown in Fig. 2(b). The new peak is localized at $q_{x} \sim Q_{x}$, when it appears. With further increasing $2 \Delta$, the gap mode extends to wider $q_{x}$ region, as shown in Fig. 2(c). The peak position of the gap mode shifts to higher energy when $q_{x}$ is away from $\boldsymbol{Q}$. The original peak along the phonon dispersion is smeared above the gap mode. When $2 \Delta$ becomes larger compared with the phonon dispersion, the original peak at the phonon dispersion vanishes, and the peak of the gap mode further grows up. To see this $\Delta$-dependence clearly, we show the $\omega$-profile of $S(\boldsymbol{q}, \omega)$ at $q_{x}=0.55 \pi$ in Fig. 3. The profiles at other $q_{x}$ also show the similar $\Delta$-dependence, while characteristic $\Delta$ value is changed. It is also noted that the lower energy tail of the peak is suppressed by increasing $\Delta$.

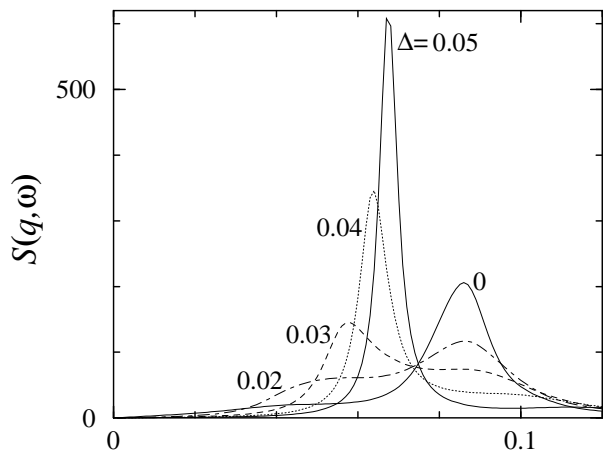

$\omega$

Fig. 3. Profiles of the phonon spectral function $S(\boldsymbol{q}, \omega)$ at $\boldsymbol{q}=$ $\pi(0.55,0,0)$ for $\Delta=0,0.02,0.03,0.04$ and 0.05 in the isotropic $s$-wave case.

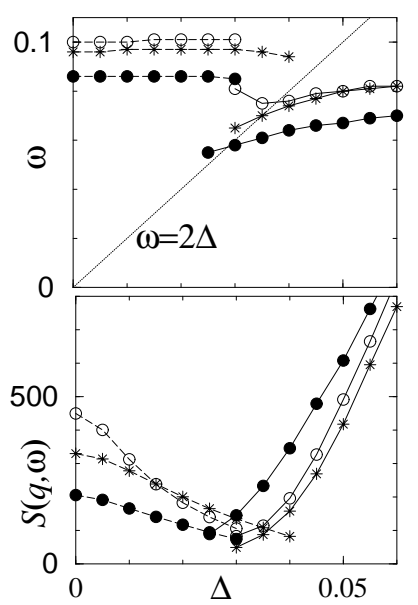

Fig. 4. $\Delta$-dependence of the peak energy (upper panel) and the peak intensity (lower panel) for $q_{x} / \pi=0.50$ (०), $0.55(\bullet), 0.60$ $(*)$. The points connected by solid lines correspond to the peak of the gap mode. Dashed lines are for the peak of the original phonon dispersion. We also show the line $\omega=2 \Delta$.

In Fig. 4, we plot the $\Delta$-dependence of the peak energy and the peak intensity. We also show the cases $q_{x}=0.50 \pi$ and $0.60 \pi$ in addition to the case $q_{x}=0.55 \pi$ in order to see the $q_{x}$-dependence. The peak intensity of the original phonon dispersion at each $q_{x}$ is monotonically decreased with increasing $\Delta$, while the peak energy at the phonon dispersion is not changed. When $2 \Delta$ approaches the phonon dispersion, these original peaks are smeared, and the new peak of the gap mode appears at lower energy. It is seen as if the new peak first appears at finite $\omega$. If we could exclude any contributions of peak broadening, we might observe new peak at $\omega \sim 2 \Delta$ until the lower $\Delta$ case near $\Delta \sim 0 .{ }^{11)}$ However, this new peak is not observed in the lower $\Delta$ case, since the peak intensity is rapidly decreased on lowering $\Delta$ and this peak is completely smeared out by the effects of peak broadening. Then, new peak appears at finite energy in our results.

Compared with the cases $q_{x}=0.5 \pi$ and $0.6 \pi$, the new peak appears from smaller $\Delta$ at $q_{x}=0.55 \pi$, since the phonon dispersion is located in lower energy at $q_{x}=$ $0.55 \pi$. The increasing rate of the new peak's energy on 
raising $\Delta$ is much weaker than the relation $\omega \sim 2 \Delta$. At larger $\Delta$, the peak energy is apparently smaller than $2 \Delta$, and it seems to reduce to an $\omega$-independent value which is smaller than the original phonon dispersion. With increasing $\Delta$, the new peak becomes sharper and it has larger intensity. Along the dispersion for $q_{x}<Q_{x}$, both the original peak and the new peak are broad and overlapped when new peak appears, as shown in Fig. 2(c). Because of the overlap, the energy of the new peak at $q_{x}=0.5 \pi$ in Fig. 4 is seen as if it appears at larger $\omega$ and decreases with increasing $\Delta$ at first.

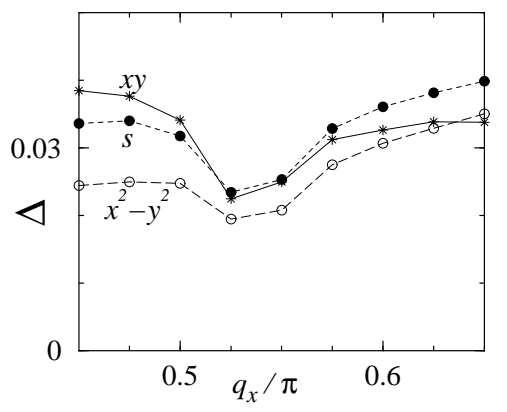

Fig. 5. $q_{x}$-dependence of the crossover $\Delta$, where peak intensity of the gap mode becomes larger than that of the original phonon peak, in the isotropic $s$-wave case $(\bullet)$, the $s_{x^{2}-y^{2}}$ case (०), and the $s_{x y}$ case $(*)$.

In Fig. 5, we plot the $q_{x}$-dependence of the crossover $\Delta$, where the peak intensity of the gap mode becomes larger than that of the original phonon peak. Above the line of the figure at each $q_{x}$, the new peak of the gap mode is eminent. The crossover $\Delta$ is low near $q_{x} \sim Q_{x}$, and increases as $q_{x}$ is away from $Q_{x}$.

The $\Delta$-dependence of the phonon profile in Fig. 3 nicely reproduces the experimental data on $\left.\mathrm{YNi}_{2} \mathrm{~B}_{2} \mathrm{C} .{ }^{3-5}\right)$ There, the $\Delta$-dependence is the temperature dependence $\Delta(T)$ or the magnetic field dependence $\Delta(H)$. In the experimental data, the energy of the new peak is close to $2 \Delta$, but the $T$-dependence of the peak energy $\omega$ is weaker than the relation $\omega=2 \Delta(T)$. This $\Delta$-dependence of the peak energy is consistent with our results. The peak appears at the energy near $2 \Delta$, when $2 \Delta$ approaches the phonon dispersion. When $\Delta$ further increases, the increase of the peak energy is weaker than the relation $\omega=2 \Delta$, as shown in Fig. 4. The experiment on $\mathrm{LuNi}_{2} \mathrm{~B}_{2} \mathrm{C}$ reports that the phonon peak, which is shifted to lower energy, becomes sharp in the superconducting state. $^{7)}$ It is consistent to our result.

\subsection{Anisotropic s-wave and $d_{x^{2}-y^{2}}$-wave superconduct- ing states}

In this subsection, we consider the anisotropic $s$-wave cases $s_{x^{2}-y^{2}}$ and $s_{x y}$ in order to clarify the effect of the superconducting gap suppression around the node points. While they have low energy excitations near nodes, we see that $S(\boldsymbol{q}, \omega)$ shows qualitatively same behavior as in the isotropic $s$-wave case presented in the previous subsection. That is, no drastic changes occur on phonon modes by their anisotropy. However, they show quantitatively different behaviors in the $\Delta$-dependence and in the $q_{x}$-dependence. We discuss these points in this subsection.

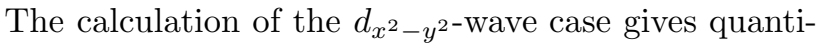
tatively almost the same $S(\boldsymbol{q}, \omega)$ as that of the $s_{x^{2}-y^{2}}$ case. This is because the nesting translation $\boldsymbol{Q}$ does not change the sign of the pairing function $\phi_{d_{x^{2}-y^{2}}}(\boldsymbol{k})$ as shown in Fig. 1(c), and $\left|\phi_{d_{x^{2}-y^{2}}}(\boldsymbol{k})\right|$ has the same gap amplitude as $\left|\phi_{s_{x^{2}-y^{2}}}(\boldsymbol{k})\right|$.
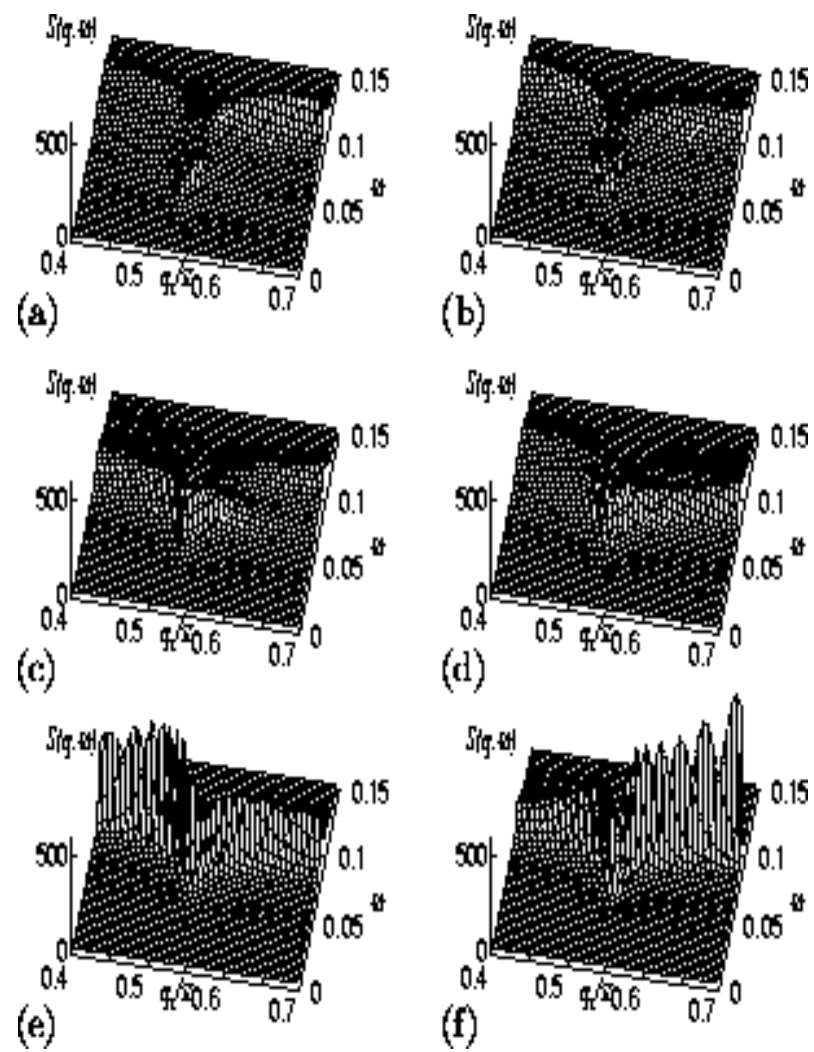

Fig. 6. Phonon spectral function $S\left(\boldsymbol{q}=\left(q_{x}, 0,0\right), \omega\right)$ in the range $0.4 \leq q_{x} / \pi \leq 0.7$ for the anisotropic $s$-wave cases. In the $s_{x^{2}-y^{2}}$ case, $\Delta=0.020$ (a), 0.030 (c) and 0.045 (e). In the $s_{x y}$ case, $\Delta=0.025(\mathrm{~b}), 0.035(\mathrm{~d})$ and $0.050(\mathrm{f})$. The $d_{x^{2}-y^{2}}$-wave case gives the same structure as that of the $s_{x^{2}-y^{2}}$ case.

In Fig. 6, we present some typical behaviors of $S(\boldsymbol{q}, \omega)$ in the $s_{x^{2}-y^{2}}$ case and the $s_{x y}$ case. They show similar behavior as in the isotropic $s$-wave case in Fig. 2. When $2 \Delta$ approaches the minimum of the phonon dispersion, a new peak of the gap mode appears at $\boldsymbol{q}=\boldsymbol{Q}[(\mathrm{a})$ and (b)]. With increasing $\Delta$, the new peak extends to the wide $q_{x}$-range [(c) and (d)]. When $\Delta$ further increases, the original peak at the phonon dispersion is completely smeared out, and the peak of the gap mode becomes sharper [(e) and (f)]. The $\Delta$-dependence of the peak energy and intensity is plotted in Fig. 7. The effect of the gap anisotropy appears at the onset $\Delta$ of the new peak and at the $q_{x}$-dependence of the peak intensity. As for the onset $\Delta$, we compare Figs. 4 and 7 . In the case of the anisotropic pairings, the superconducting gap is enhanced or suppressed by the factor $\phi(\boldsymbol{k})$ in $\Delta_{\boldsymbol{k}}=$ $\Delta(T) \phi(\boldsymbol{k})$. In the $s_{x^{2}-y^{2}}$ case, $\phi_{s_{x^{2}-y^{2}}}(\boldsymbol{k})$ is larger than 1 along the flat Fermi surface $0 \leq \theta<30^{\circ}$ as seen in Fig. 1 (b). At $\theta=0, \phi_{s_{x^{2}-y^{2}}}(\boldsymbol{k})$ has maximum 1.65. Then, we also plot the line $\omega=2 \max \left(\Delta_{\boldsymbol{k}}\right)=3.3 \Delta$ in addition to the line $\omega=2 \Delta$ in Fig. 7. In Fig. 7(a), the onset $\Delta$ of 
the new peak is lower than that of the isotropic $s$-wave case, but it is larger than the relation $\omega=3.3 \Delta$. That is, since the effect of $\phi_{s_{x^{2}-y^{2}}}(\boldsymbol{k})$ is averaged on the flat Fermi surface, the enhancement factor is larger than 1, but smaller than 1.65. Compared with the $s_{x^{2}-y^{2}}$ case, the onset $\Delta$ is larger in the $s_{x y}$ case, since the average of the factor $\phi_{s_{x y}}(\boldsymbol{k})$ on the flat Fermi surface is smaller.

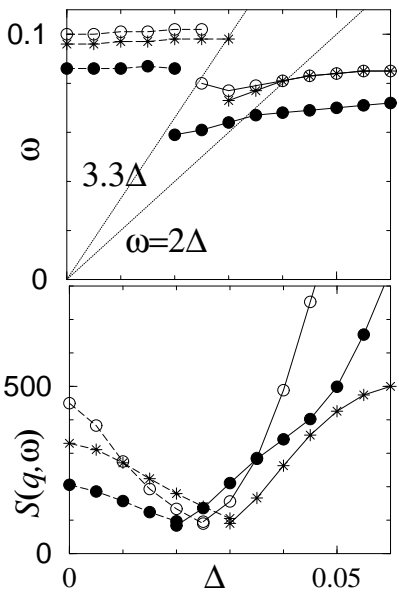

(a)

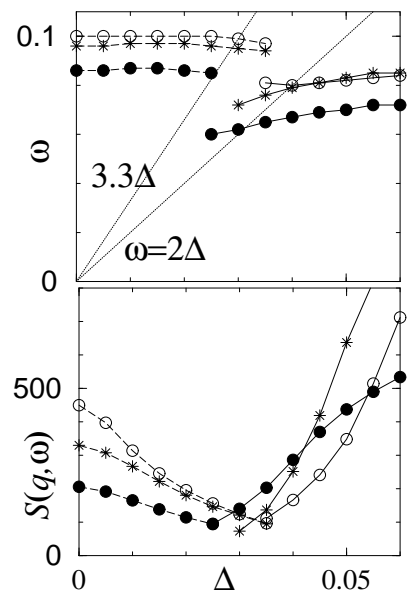

(b)
Fig. 7. $\Delta$-dependence of the peak energy (upper panels) and the peak intensity (lower panels) for $q_{x} / \pi=0.50(\circ), 0.55(\bullet), 0.60$ $(*)$ in the $s_{x^{2}-y^{2}}$ case (a) and in the $s_{x y}$-case (b). The points connected by solid lines correspond to the peak of the gap mode. Dashed lines are for the peak of the original phonon dispersion. We also show the lines $\omega=2 \Delta$ and $\omega=3.3 \Delta$.

As for the $q_{x}$-dependence of the peak intensity, the intensity of the gap mode in Fig. 6 is enhanced for $q_{x}<$ $Q_{x}\left(q_{x}>Q_{x}\right)$ in the $s_{x^{2}-y^{2}}$ case $\left(s_{x y}\right.$ case) compared with the isotropic $s$-wave case. The gap mode eminently extends toward $q_{x}<Q_{x}\left(q_{x}>Q_{x}\right)$ in Fig. 6(c) (Fig. $6(\mathrm{~d}))$. These characteristics are also recognized in Fig. 7 , where the peak intensity of the gap mode at $q_{x}=0.5 \pi$ $(0.6 \pi)$ is largely enhanced, and in Fig. 5, where crossover $\Delta$ is lower for $q_{x}<Q_{x}\left(q_{x}>Q_{x}\right)$. This difference comes from the $\boldsymbol{k}$-dependence of $\phi(\boldsymbol{k})$ along the perpendicular direction to the flat Fermi surface. We explain it by the $k_{x}$-dependence near the flat Fermi surface at $k_{x}=0.275 \pi$ in Fig. 1(a). In the $s_{x^{2}-y^{2}}$ case, the factor $\phi_{s_{x^{2}-y^{2}}}(\boldsymbol{k})$ is enhanced when $k_{x}$ is decreased because of the term $\cos k_{x}$ in $\phi_{s_{x^{2}-y^{2}}}(\boldsymbol{k})$. Then, for the smaller $q_{x}\left(\sim 2 k_{x}\right)$ than the nesting vector $Q_{x}$, the superconducting gap is enhanced, and the intensity of the gap mode becomes eminent. On the other hand, $\phi_{s_{x y}}(\boldsymbol{k})$ is enhanced when $k_{x}$ is increased because of the factor $\sin k_{x}$ in $\phi_{s_{x y}}(\boldsymbol{k})$. Then, for larger $q_{x}\left(\sim 2 k_{x}\right)$ than $Q_{x}$, the gap mode is eminent.

\section{$3.4 d_{x y}$-wave and $p_{x}$-wave superconducting states}

In this subsection, we examine the effect coming from the sign change of the pairing function by the nesting translation. The $d_{x y}$-wave case has the same superconducting gap as that of the $s_{x y}$ case. But, the sign of the pairing function $\phi_{d_{x y}}(\boldsymbol{k})$ is changed by the nesting translation $\boldsymbol{Q}$ as shown in Fig. 1(d). Then, the phonon behavior in the $d_{x y}$-wave case is qualitatively different from the $s_{x y}$ case. It is because $\operatorname{Re}\left\{\Delta_{\boldsymbol{k}+\boldsymbol{q}} \Delta_{\boldsymbol{k}}^{*}\right\}$ of $\chi_{n n}(\boldsymbol{q}, \omega)$ in eq. (2) becomes negative at $\boldsymbol{q} \sim \boldsymbol{Q}$ when the sign change occurs. ${ }^{20)}$ The $p_{x}$-wave case also shows the same behavior due to the sign change of $\phi_{p_{x}}(\boldsymbol{k})$ through the nesting translation.
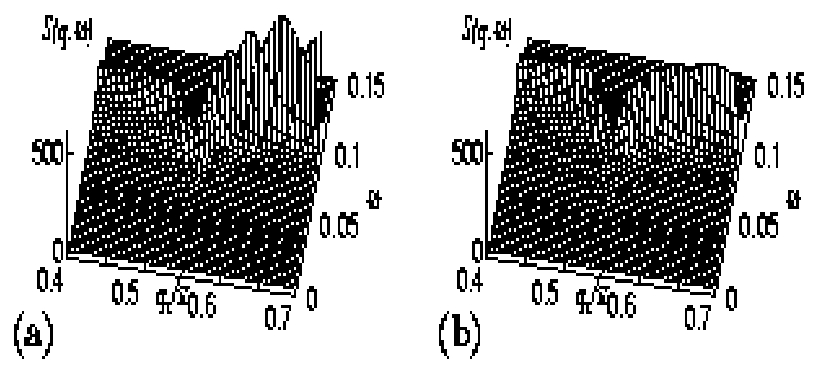

Fig. 8. Phonon spectral function $S\left(\boldsymbol{q}=\left(q_{x}, 0,0\right), \omega\right)$ in the range $0.4 \leq q_{x} / \pi \leq 0.7$ for the $d_{x y}$-wave case (a) and the $p_{x}$-wave case (b). $\Delta=0.030$.

Figure 8 shows the typical behavior of $S(\boldsymbol{q}, \omega)$ in the $d_{x y}$-wave (a) and the $p_{x}$-wave (b) cases. There, the new peak of the gap mode does not appear. By the effect of $\Delta$, the peak intensity of the original phonon mode is modified. But, the peak energy along the phonon dispersion is almost unchanged. To see this $\Delta$-dependence, we show the $\omega$-profile of $S(\boldsymbol{q}, \omega)$ at $q_{x}=0.55 \pi$ for the $d_{x y}$-wave case in Fig. 9 . With increasing $\Delta$, the low energy tail is gradually suppressed. After $2 \Delta$ exceeds the phonon dispersion, the peak intensity becomes sharper and the intensity is enhanced with increasing $\Delta$. To see the $q_{x}$-dependence of this behavior, we plot the $\Delta$-dependence of the peak intensity at $q_{x} / \pi=0.50$, $0.55,0.60$ in Fig. 10. At low $\Delta$, the peak intensity at $q_{x}=0.50 \pi(0.60 \pi)$ is suppressed (enhanced) with increasing $\Delta$, as also shown in Fig. 8. This $q_{x}$-dependence comes from the $k_{x}$-dependence of the pairing functions $\left|\phi_{d_{x y}}(\boldsymbol{k})\right|$ and $\left|\phi_{p_{x}}(\boldsymbol{k})\right|$ near the flat Fermi surface at $k_{x}=0.275 \pi$. There, $\left|\phi_{d_{x y}}(\boldsymbol{k})\right|$ and $\left|\phi_{p_{x}}(\boldsymbol{k})\right|$ is enhanced with increasing $k_{x}$ because of the factor $\sin k_{x}$ in the pairing functions. Then, for larger $q_{x}\left(\sim 2 k_{x}\right)$ than $Q_{x}$, the superconducting gap is stronger, and the peak intensity is enhanced.

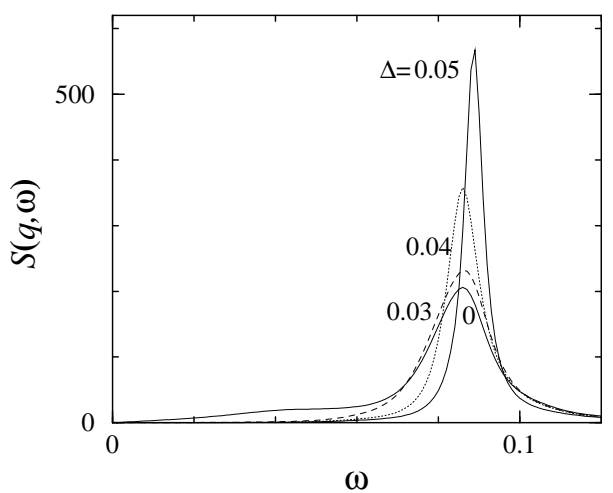

Fig. 9. Profiles of the phonon spectral function $S(\boldsymbol{q}, \omega)$ at $\boldsymbol{q}=$ $\pi(0.55,0,0)$ for $\Delta=0,0.03,0.04$ and 0.05 in the $d_{x y}$-wave case.

\section{Suppression of phonon softening}

When $\omega_{\boldsymbol{q}}$ is enough low, the phonon dispersion approaches zero energy at $\boldsymbol{q}=\boldsymbol{Q}$ on lowering temperature 


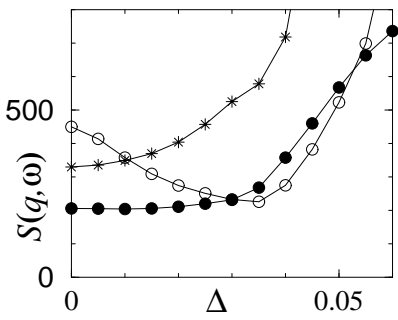

(a)

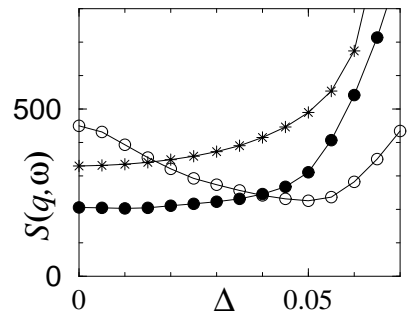

(b)
Fig. 10. $\Delta$-dependence of the peak intensity for $q_{x} / \pi=0.50$ (o), $0.55(\bullet), 0.60(*)$ in the $d_{x y}$-wave case (a) and the $p_{x}$-wave case (b).

due to the Kohn anomaly. This phonon softening causes the second order transition to the CDW states. It is suggested that this softening is suppressed by the onset of superconductivity. ${ }^{11)}$ To study this phenomenon, we consider the phonon properties in the case $\omega_{\boldsymbol{q}}=0.117$. The other parameters are unchanged.

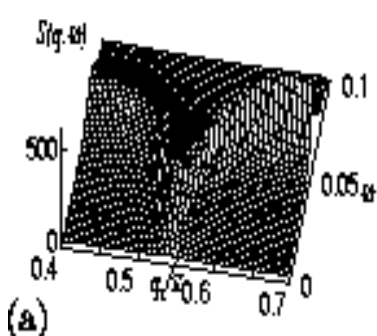

(a)
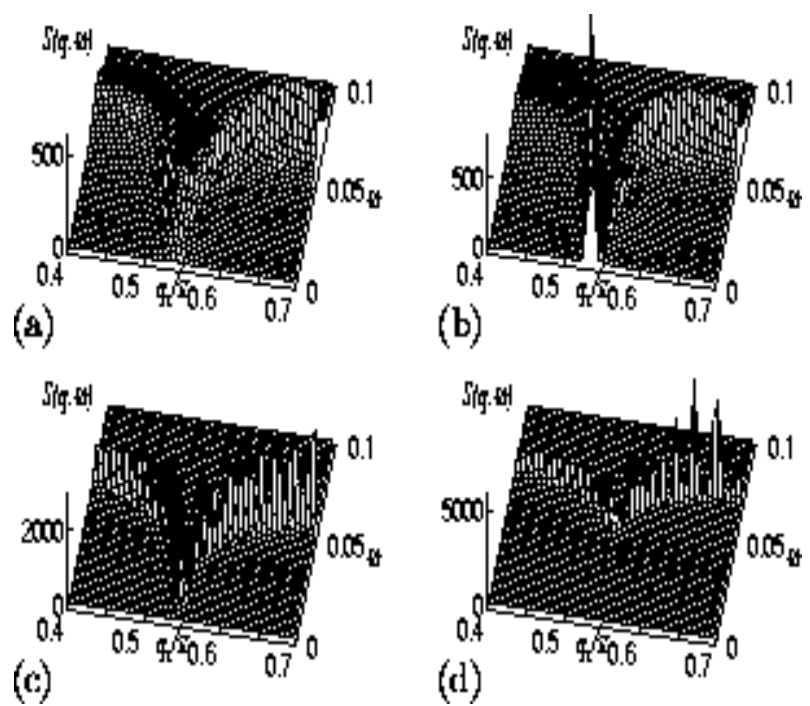

Fig. 11. Phonon spectral function $S\left(\boldsymbol{q}=\left(q_{x}, 0,0\right), \omega\right)$ in the range $0.4 \leq q_{x} / \pi \leq 0.7$ for $\omega_{\boldsymbol{q}}=0.117$. In the normal state $(\Delta=0)$, $T=0.050$ (a) and 0.005 (b). In the superconducting state of the $s_{x y}$-case (c) and the $d_{x y}$-wave case $(\mathrm{d}), \Delta=0.05$ and $T=0.005$.

Figure 11 shows $S(\boldsymbol{q}, \omega)$ in this case. At high temperature $T=0.050(\mathrm{a})$, the phonon dispersion is located at finite energy. With decreasing temperature, the phonon dispersion is shifted to lower energy within the narrow region at $\boldsymbol{q}=\boldsymbol{Q}$. At low temperature $T=0.005$ (b) in the normal state, the minimum of the dispersion touches zero energy, suggesting CDW transition. However, in the superconducting state, the phonon dispersion remains at finite energy, meaning the suppression of the CDW transition. Figure $11(\mathrm{c})$ is for the $s_{x y}$ case. We obtain this type phonon behavior in the isotropic and anisotropic $s$-wave cases, and the $d_{x^{2}-y^{2}}$-wave case, i.e., in the case when the sign of the pairing function is not changed by the nesting translation. When the sign is changed by the nesting, such as the case $d_{x y}$-wave and $p_{x}$-wave cases, the phonon dispersion is further shifted to higher energy. We show $S(\boldsymbol{q}, \omega)$ of the $d_{x y}$-wave case in Fig. $11(\mathrm{~d})$. It is noted that these change of the phonon dispersion occurs only near $\boldsymbol{q}=\boldsymbol{Q}$. The dispersion is not largely changed far away from $\boldsymbol{Q}$, e.g., at $q_{x}=0.4 \pi$ or $0.7 \pi$ in Fig. 11 .

We plot the temperature dependence of the minimum energy of the phonon dispersion in Fig. 12, where the phonon dispersion is identified by the peak energy. In the normal state, the minimum energy $\omega$ monotonically decreases on lowering temperature. At $T \sim 0.005$, the CDW transition occurs by the softening $\omega \rightarrow 0$. On the other hand, the softening stops in the superconducting state. Then, the second order transition to the CDW state does not occur. The minimum $\omega$ shows weak temperature dependence below $T_{c}$ in the $s$-wave cases

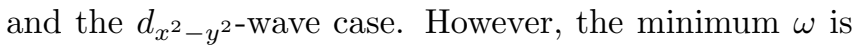
largely enhanced just below $T_{c}$ and it saturates at low temperature in the $d_{x y}$-wave and the $p_{x}$-wave cases, i.e., in the case when the pairing function changes the sign through the nesting translation. In Fig. 12, $T_{c}$ is different due to the factor $\phi(\boldsymbol{k})$, while we set $\Delta(T=0)=0.05$ in each pairing case.

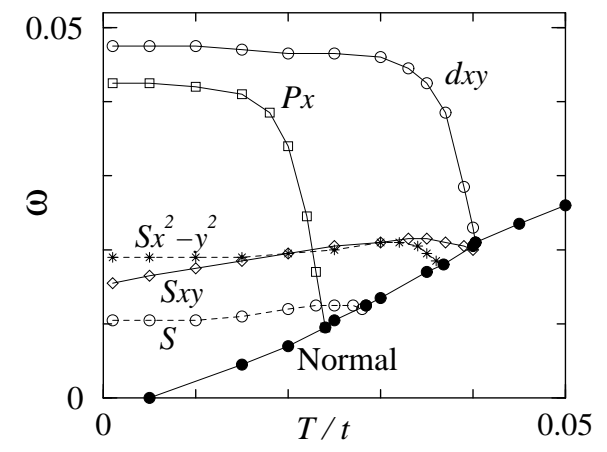

Fig. 12. Temperature dependence of the minimum energy of the phonon dispersion for $\omega_{\boldsymbol{q}}=0.117$ in the normal state $(\Delta=$ $0)$ and the superconducting state with $\Delta(T=0)=0.05$. In the superconducting state, we present the isotropic $s$-wave, the anisotropic $s$-wave $s_{x^{2}-y^{2}}$ and $s_{x y}$, the $d_{x y}$-wave and the $p_{x^{-}}$ wave cases. The points for the $d_{x^{2}-y^{2}}$-wave is the same as the $s_{x^{2}-y^{2}}$ case.

The amplitude of the superconducting gap is also controlled by the external magnetic field. In the case when the CDW transition is suppressed by superconductivity, the CDW state is recovered if we destroy the superconductivity by applying the magnetic field. This prediction is testable for appropriate superconductors, possibly $\mathrm{YNi}_{2} \mathrm{~B}_{2} \mathrm{C}$.

\section{Summary and discussions}

By extending the previous theories ${ }^{8-11)}$ on the appearance of the new phonon mode below $T_{\mathrm{c}}$ for isotropic $s$ wave superconductors, we have investigated the detailed features of the dispersion relation for this new phonon gap mode in light of various possible anisotropic pairings. It is demonstrated that observation of this dispersive gap mode can be a useful spectroscopic tool for sorting out some of the possible pairing states. With increasing the amplitude of the superconducting gap $\Delta$, this new peak of the gap mode becomes sharp and the original phonon peak is smeared. We have estimated the $\Delta$-dependence of the dispersion and the intensity of the peak. The effect of the gap anisotropy is classified 
to two cases; (i) the case when the sign of the pairing function is not changed through the nesting translation, and (ii) the case when the sign is changed. In the former case (i), the gap anisotropy modifies the phonon spectral function quantitatively. The anisotropy effects appear at the onset $\Delta$ of the gap mode and at the $q_{x}$-dependence of the peak intensity, reflecting local amplitude of the superconducting gap at the Fermi surface region related to the nesting translation. In the latter case (ii), the new peak of the gap mode does not appear. There, for larger $\Delta$, the original phonon peak becomes sharp without large shift of the peak energy. While we obtain these properties by analyzing example models for the Fermi surface and pairing functions, we can expect that these qualitative results coming from the superconducting gap anisotropy are independent of the model, because of the generality of their origin.

In the experimental data on $\mathrm{YNi}_{2} \mathrm{~B}_{2} \mathrm{C}$, the eminent dispersive new peak of the gap mode is observed around $\boldsymbol{q}=\boldsymbol{Q} \cdot{ }^{3-5)}$ This behavior of the gap mode is qualitatively consistent to our numerical results. The phonon peak on $\mathrm{LuNi}_{2} \mathrm{~B}_{2} \mathrm{C}$ is shifted to lower energy in the superconducting state. ${ }^{7}$ ) Then, in these materials, we can exclude the pairing symmetry of the case (ii), for example, $d_{x y}$-wave. That is, we can conclude that the pairing function has the same sign at the flat Fermi surface related to the nesting.

Further identification of the pairing symmetry among isotropic $s$-wave, anisotropic $s$-wave and $d_{x^{2}-y^{2} \text {-wave is }}$ difficult, because it needs quantitative considerations. But, we give some discussions here. One of the key points to identify the pairing symmetry is the $q_{x}$-dependence of the peak intensity. In the data on $\mathrm{YNi}_{2} \mathrm{~B}_{2} \mathrm{C}$, the peak intensity is larger for $q_{x}<Q_{x}{ }^{3)}$ Following our analysis, this suggests that the gap anisotropy of the type $\left|\cos k_{x}-\cos k_{y}\right|$ is preferable. The second point is that the superconducting gap largely opens at the flat Fermi surface related to the nesting, when the eminent gap mode appears. The contribution of the superconducting gap affects on the phonon as an average along the flat Fermi surface. In our calculation, the average of $\phi_{s_{x y}}(\boldsymbol{k})$ is smaller than that of $\phi_{s_{x^{2}-y^{2}}}(\boldsymbol{k})$. The former has a node at the flat part of the Fermi surface. Since the flat part is long in our model of the Fermi surface, the both averages do not show large difference. How- ever, if the flat part is shorter, the average of the gap is smaller in the case when the node of the gap anisotropy is located at the flat part. In this case, we cannot expect the eminent gap mode. To obtain decisive conclusion on these above-mentioned points, we need to establish the knowledge about the detailed shape of the Fermi surface and the nesting properties.

Note added: Recently, a similar neutron experiment has been reported on $\mathrm{ErNi}_{2} \mathrm{~B}_{2} \mathrm{C}$ by Kawano-Furukawa et al., showing the gap mode below $T_{\mathrm{c}}{ }^{21}$ )

\section{Acknowledgments}

We would like to thank H. Yoshizawa for helpful discussions.

1) W. Kohn: Phys. Rev. Lett. 2 (1959) 393.

2) R. Sooryakumar and M.V. Klein: Phys. Rev. Lett. 45 (1980) 660.

3) H. Kawano, H. Yoshizawa, H. Takeya and K. Kadowaki: Phys. Rev. Lett. 77 (1996) 4628.

4) H. Kawano, H. Yoshizawa, H. Takeya and K. Kadowaki: Physica C 282-287 (1997) 1055.

5) H. Kawano, H. Yoshizawa, H. Takeya and K. Kadowaki: Physica B 241-243 (1998) 874.

6) S.B. Dugdale, M.A. Alam, I. Wilkinson, R.J. Hughes, I.R. Fisher, P.C. Canfield, T. Jarlborg and G. Santi: Phys. Rev. Lett. 83 (1999) 4824.

7) C. Stassis, M. Bullock, J. Zarestky, P. Canfield, A.I. Goldman, G. Shirane and S.M. Shapiro: Phys. Rev. B 55 (1997) R8678.

8) C.A. Balseiro and L.M. Falicov: Phys. Rev. Lett. 45 (1980) 662 .

9) P.B. Littlewood and C.M. Varma: Phys. Rev. Lett. 47 (1981) 811.

10) P.B. Allen, V.N. Kostur, N. Takesue and G. Shirane: Phys. Rev. B 56 (1997) 5552.

11) H-Y. Kee and C.M. Varma: Phys. Rev. Lett. 79 (1997) 4250.

12) W.E. Pickett and D.J. Singh: Phys. Rev. Lett. 72 (1994) 3702.

13) L. Mattheiss: Phys. Rev. B 49 (1994) 13279.

14) R. Coehoorm: Physica (Amsterdam) 228 C (1994) 331.

15) J.I. Lee, T.S. Zhao, I.G. Kim, B.I. Min and S.J. Youn: Phys. Rev. B 50 (1994) 4030.

16) T. Yokoya, T. Kiss, T. Watanabe, S. Shin, M. Nohara, H. Takagi and T. Oguchi: Phys. Rev. Lett. 85 (2000) 4952.

17) M. Nohara, M. Isshiki, F. Sakai and H. Takagi: J. Phys. Soc. Jpn 68 (1999) 1078

18) M. Nohara, H. Suzuki, N. Mangkorntong and H. Takagi: Physica C (Amsterdam) 341-348 (2000) 2177.

19) K.Izawa, A. Shibata, Y. Matsuda, Y. Kato, H. Takeya, K. Hirata, C.J. van der Beek and M. Konczykowski: Phys. Rev. Lett. 86 (2001) 1327.

20) H-Y. Kee and C.M. Varma: Phys. Rev. B 58 (1998) 15035.

21) H. Kawano-Furukawa, H. Yoshizawa, H. Takeya and K. Kadowaki: Phys. Rev. B 66 (2002) 212503. 